\title{
BIEMBEDDINGS OF LATIN SQUARES AND HAMILTONIAN DECOMPOSITIONS
}

\author{
M. J. GRANNELL, T. S. GRIGGS \\ Department of Pure Mathematics, The Open University, Walton Hall, Milton Keynes MK7 6AA, \\ United Kingdom \\ e-mail:m.j.grannell@open.ac.uk,t.s.griggs@open.ac.uk \\ and M. KNOR \\ Department of Mathematics, Faculty of Civil Engineering, Slovak University of Technology, \\ Radlinského 11,813 68 Bratislava, Slovakia \\ e-mail:knor@vox.svf.stuba.sk
}

(Received 22 April, 2003; accepted 22 January, 2004)

\begin{abstract}
Face 2-colourable triangulations of complete tripartite graphs $K_{n, n, n}$ correspond to biembeddings of Latin squares. Up to isomorphism, we give all such embeddings for $n=3,4,5$ and 6 , and we summarize the corresponding results for $n=7$. Closely related to these are Hamiltonian decompositions of complete bipartite directed graphs $K_{n, n}^{*}$, and we also give computational results for these in the cases $n=3,4,5$ and 6 .
\end{abstract}

2000 Mathematics Subject Classification. 05B15, 05C10.

1. Introduction. A number of recent papers $[2,5,6]$ have dealt with biembeddings of pairs of Steiner triple systems (STSs) in both orientable and nonorientable surfaces. Such a biembedding corresponds to a face 2-colourable triangulation of a complete graph $K_{n}$. The vertices of the graph form the points of the Steiner triple systems and the triangular faces in each of the two colour classes respectively form the triples of each system. We here recall that an $\operatorname{STS}(n)$ may be formally defined as an ordered pair $(V, \mathcal{B})$, where $V$ is an $n$-element set (the points) and $\mathcal{B}$ is a set of 3-element subsets of $V$ (the triples), such that every 2-element subset of $V$ appears in precisely one triple. Such systems are known to exist if and only if $n \equiv 1$ or $3(\bmod 6)$. We say that two $\operatorname{STS}(n) \mathrm{s}$ are biembedded in a surface if there is a face 2-colourable triangulation of $K_{n}$ in which the face sets forming the two colour classes give copies of the two systems. We will take the colour classes of face 2-colourable embeddings to be black and white.

One may consider embeddings which involve other types of combinatorial design. Embeddings of complete tripartite graphs are discussed in [7, 11] and form a useful tool in recursive constructions for biembeddings of Steiner triple systems. A face 2colourable triangulation of the complete tripartite graph $K_{n, n, n}$ may be considered as a biembedding of a pair of transversal designs $\mathrm{TD}(3, n)$; such a design comprises an ordered triple $(V, \mathcal{G}, \mathcal{B})$, where $V$ is a $3 n$-element set (the points), $\mathcal{G}$ is a partition of $V$ into three disjoint sets (the groups) each of cardinality $n$, and $\mathcal{B}$ is a set of 3element subsets of $V$ (the triples), such that every unordered pair of elements from $V$ is either contained in precisely one triple or one group, but not both. As with STSs, the vertices of the embedded graph $K_{n, n, n}$ form the points of the designs, the tripartition 
determines the groups, and the faces in each colour class form the triples of each design. The connection with Latin squares is that a $\operatorname{TD}(3, n)$ determines a main class of Latin squares by assigning the three groups of the design as the identifiers of the rows, columns and entries (in any one of the six possible orders) of the Latin square. Thus a face 2-colourable triangulation of $K_{n, n, n}$ may be considered as a biembedding of two Latin squares.

Before proceeding, we here review some basic aspects of notation and terminology which are important for our discussion. For further details we refer the reader to texts such as $[3,9, \mathbf{1 0}]$. Two Latin squares of the same order $n$ will be regarded as isomorphic if they belong to the same main class, i.e. if there exist three bijections mapping the row, column and entry identifiers of the first square to those of the second (not necessarily in the same order) which map the first square to the second. We assume that the reader is familiar with the description of topological embeddings by means of rotation schemes. Our embeddings will always be in surfaces rather than pseudosurfaces (the latter result from surfaces by repeating a finite number of times the operation of identifying a finite number of points on a surface). Equivalently, in the description of an embedding by means of a rotation scheme, the rotation about each vertex comprises a single cycle. In Section 4 we make use of voltage graphs to construct certain embeddings. When discussing isomorphisms and automorphisms of embeddings, we allow in the orientable case mappings which reverse the orientation and, in the case of face 2-colourability, mappings which reverse the two colour classes.

An embedding of a graph $G$ is said to be regular if and only if for every two flags, i.e. triples $\left(v_{1}, e_{1}, f_{1}\right)$ and $\left(v_{2}, e_{2}, f_{2}\right)$, where $e_{i}$ is an edge incident with the vertex $v_{i}$ and the face $f_{i}$, there exists an automorphism of the embedding which maps $v_{1}$ to $v_{2}, e_{1}$ to $e_{2}$ and $f_{1}$ to $f_{2}$. This definition of regularity is equivalent to requiring that the automorphism group of the embedding be as large as possible. Thus, in the case $G=K_{n, n, n}$, an embedding is regular if and only if its automorphism group has order $12 n^{2}$; there being $3 n^{2}$ edges each of which is incident with two vertices and two faces.

We point out that the definition of regularity varies somewhat between authors; see $[1$, p. 36] for a discussion of the terminology. The definition of regularity given above, called by some authors reflexive regularity, requires the admission of automorphisms which reverse the orientation of the surface in the orientable case. However, many authors require that any global orientation of the surface is preserved and this means that their regular embeddings may be less symmetric.

Our first observation is easily proved but, possibly, surprising.

\section{Proposition 1. A triangulation of $K_{n, n, n}$ is orientable if and only if it is face 2- colourable.}

Proof. Suppose that $K_{n, n, n}$ has tripartition $\{A, B, C\}$. If an orientable embedding is given, then triangles with clockwise orientation $(A, B, C)$ may be coloured black and those with clockwise orientation $(A, C, B)$ may be coloured white. Conversely, suppose that a face 2-colourable triangulation is given. If a black triangle of the embedding with vertices $a \in A, b \in B, c \in C$ is oriented, say clockwise, as $(A, B, C)$, then all black triangles incident with $a$ also have clockwise orientation $(A, B, C)$, while the white triangles incident with $a$ have orientation $(A, C, B)$. Since the vertices of these triangles span $B \cup C$, all remaining black triangles have clockwise orientation $(A, B, C)$ and all remaining white triangles have clockwise orientation $(A, C, B)$. It follows that the rotation scheme for the embedding satisfies Ringel's rule $R^{*}$ (see $[10$, p. 28]) and therefore represents an orientable embedding. 
Our second observation relates face 2-colourable embeddings of $K_{n, n, n}$ to certain orientable embeddings of $K_{n, n}$.

Proposition 2. Given an orientable embedding of $K_{n, n}$ in which all face boundaries form Hamiltonian cycles, a face 2-colourable triangulation of $K_{n, n, n}$ may be constructed by inserting a new vertex into the interior of each face and joining it by new non-intersecting edges to all the vertices on the boundary of that face. Conversely, given a face 2-colourable triangulation of $K_{n, n, n}$ with tripartition $\{A, B, C\}$, by deleting all vertices in one of the sets $A, B$ or $C$, together with all (open) incident edges, we may form an orientable embedding of $K_{n, n}$ in which all the face boundaries form Hamiltonian cycles.

Proof. Given an orientable embedding of $K_{n, n}$ in which all the face boundaries form Hamiltonian cycles, by counting edges, the number $N$ of such faces is given by $2 n \times N=2 n^{2}$. Thus $N=n$. If the bipartition is $\{A, B\}$ then the Hamiltonian cycles alternate points of $A$ with points of $B$. Inserting a new vertex $c$ into the interior of a face and adding the edges as described results in oriented triangles incident with $c$ which alternately have the forms $\left(c, a_{i}, b_{j}\right)$ and $\left(c, b_{k}, a_{l}\right)$ where $a_{i}, a_{l} \in A$ and $b_{j}, b_{k} \in B$. The former may all be coloured black and the latter white, giving a proper face 2-colouring of the resulting $K_{n, n, n}$ embedding. The converse follows immediately.

Given an orientable embedding of $K_{n, n}$ in which all the face boundaries form Hamiltonian cycles, by assigning an orientation, we obtain a decomposition of the complete bipartite directed graph $K_{n, n}^{*}$ into directed Hamiltonian cycles. For any decomposition of $K_{n, n}^{*}$ into directed Hamiltonian cycles, we may define the neighbourhood of a vertex $x$ to be the graph formed on the $n$ vertices adjacent to $x$ by joining two vertices with an edge if and only if they are both adjacent to $x$ in the same Hamiltonian cycle. We say that a decomposition of $K_{n, n}^{*}$ into directed Hamiltonian cycles is perfect if the neighbourhood of every vertex is a single cycle. Plainly, a decomposition arising from an orientable embedding of $K_{n, n}$ is perfect. Conversely, a perfect decomposition gives an orientable embedding of $K_{n, n}$ in which all the face boundaries form Hamiltonian cycles because the neighbourhood of each point defines the rotation about that point. Thus we have established the following result.

PROPOSITION 3. There is a one-to-one correspondence between orientable embeddings of $K_{n, n}$ in which all the face boundaries form Hamiltonian cycles, and perfect decompositions of the complete bipartite directed graph $K_{n, n}^{*}$ into directed Hamiltonian cycles.

As a consequence of the above propositions, we may search for biembeddings of Latin squares either directly or by first examining decompositions of $K_{n, n}^{*}$ into directed Hamiltonian cycles and then restricting attention to those decompositions which are perfect. In an attempt to verify our computational results, we have used both approaches, and then reconciled the corresponding outputs. In making this reconciliation, account has to be taken of the fact that up to three different (i.e. nonisomorphic) $K_{n, n}$ embeddings may result from deleting in turn each of the three sets of the tripartition in an embedding of $K_{n, n, n}$. The cases of $K_{1,1,1}$ and $K_{2,2,2}$ correspond respectively to a spherical embedding of a triangle and an octahedron. In order to avoid trivial cases, throughout the remainder of this paper we assume that $n \geq 3$. 
2. Hamiltonian decompositions of $\boldsymbol{K}_{\boldsymbol{n}, \boldsymbol{n}}^{*}$. Perhaps surprisingly, no enumeration of Hamiltonian decompositions of $K_{n, n}^{*}$ for small $n$ seems to have been undertaken. This paper rectifies this deficiency for $n=3,4,5$ and 6 . We take the bipartition of $K_{n, n}^{*}$ to be $\{A, B\}$ where $|A|=|B|=n$. The points of $A$ will be denoted by $a_{i}$ and those of $B$ by $b_{i}$. Given a decomposition $D_{1}$, we may form a decomposition $D_{2}$ by reversing all the cycles; we regard $D_{1}$ and $D_{2}$ as identical decompositions. The automorphism group of the decomposition $D$ will be denoted by $\operatorname{Aut}(D)$. A directed Hamiltonian cycle such as $\left(a_{0}, b_{1}, a_{1}, b_{0}, a_{2}, b_{3}, a_{3}, b_{2}\right)$ will be given more succinctly as 01102332 ; by convention all cycles start with $a_{0}$.

The decompositions were found by an exhaustive backtracking program. Without loss of generality, it was assumed that each decomposition of $K_{n, n}^{*}$ contains the cycle $a_{0} b_{0} a_{1} b_{1} \cdots a_{n-1} b_{n-1}$. Isomorphisms between decompositions were then easily determined because of the limited number of possibilities: the cycle $a_{0} b_{0} a_{1} b_{1} \cdots a_{n-1} b_{n-1}$ can only map to one of $n$ possible $2 n$-cycles, and there are only $2 \times 2 n$ ways of defining each such mapping. The automorphism group of each decomposition was also easily found in a similar manner.

$\boldsymbol{n}=3$. Up to isomorphism, there is a unique decomposition and this is perfect. The cycles are: $001122,011220,021021$. The automorphism group has order 36; nine of these mappings preserve the bipartition and direction of the cycles, nine exchange $A$ and $B$ but preserve the direction, nine preserve the bipartition but reverse the direction of the cycles, and nine exchange $A$ and $B$ and reverse the direction.

$\boldsymbol{n}=4$. Up to isomorphism, there are four decompositions of which one is perfect. They are as follows.

1. 00112233, 01102332, 02132031,03122130. $\mid$ Aut $(D) \mid=32$, all mappings preserve the bipartition and 16 preserve direction.

2. 00112233, 01102332, 02132130, 03122031. $\mid$ Aut $(D) \mid=16$, all mappings preserve the bipartition and eight preserve direction.

3. 00112233, 01122330, 02132031, 03102132. $\mid$ Aut $(D) \mid=64,16$ mappings preserve the bipartition and directions, 16 reverse the bipartition but preserve the direction, 16 preserve the bipartition but reverse direction, and 16 reverse the bipartition and the direction. This decomposition is perfect.

4. 00112233, 01302312, 02132031, 03322110. $\mid$ Aut $(D) \mid=32$, of which 16 mappings preserve the bipartition. Each Hamiltonian cycle in this decomposition appears with its reverse so that each automorphism may be considered as both preserving and reversing the directions. We call such a decomposition reversible.

$\boldsymbol{n}=5$. Up to isomorphism, there are 14 decompositions of which four are perfect. All 14 are shown in Table 1 with the notation $\left(M ; m_{1}, m_{2}, m_{3}, m_{4}\right)$ denoting that $|A u t(D)|=M$ and that there are $m_{1}$ mappings which preserve direction and bipartition, $m_{2}$ mappings which preserve direction and reverse the bipartition, $m_{3}$ mappings which reverse direction and preserve the bipartition, and $m_{4}$ mappings which reverse direction and reverse the bipartition.

$\boldsymbol{n}=\mathbf{6}$. Up to isomorphism there are 18969 decompositions. Of these 59 are perfect and a further seven are reversible. Reversible decompositions correspond to Hamiltonian decompositions of the undirected $K_{6,6}$. The 59 perfect decompositions are given in Table 2 in the same format as those for the $n=5$ case. The seven Hamiltonian 
Table 1. All decompositions of $K_{5,5}^{*}$.

\begin{tabular}{|c|c|c|c|c|c|c|c|}
\hline 1 & 0011223344 & 0110233442 & 0213243041 & 0412203143 & 0314213240 & $(2 ; 1,0,1,0)$ & \\
\hline 2 & 0011223344 & 0110233442 & 0312243041 & 0214203143 & 0413213240 & $(2 ; 1,0,1,0)$ & \\
\hline 3 & 0011223344 & 0110233442 & 0324124031 & 0420413213 & 0221431430 & $(1 ; 1,0,0,0)$ & \\
\hline 4 & 0011223344 & 0112233440 & 0213243041 & 0314203142 & 0410213243 & $(100 ; 25,25,25,25)$ & perfect \\
\hline 5 & 0011223344 & 0112233440 & 0241302413 & 0314203142 & 0432104321 & $(20 ; 5,5,5,5)$ & \\
\hline 6 & 0011223344 & 0112233440 & 0241302413 & 0320421431 & 0421431032 & $(20 ; 5,5,5,5)$ & perfect \\
\hline 7 & 0011223344 & 0112243043 & 0214233140 & 0310213442 & 0413203241 & $(10 ; 5,0,5,0)$ & perfect \\
\hline 8 & 0011223344 & 0112243043 & 0241341320 & 0423103142 & 0332144021 & $(2 ; 1,0,1,0)$ & \\
\hline 9 & 0011223344 & 0112243043 & 0241341320 & 0442102331 & 0314214032 & $(2 ; 1,0,1,0)$ & \\
\hline 10 & 0011223344 & 0112243043 & 0241342310 & 0332144021 & 0413203142 & $(2 ; 1,0,1,0)$ & \\
\hline 11 & 0011223344 & 0113243042 & 0210342143 & 0412402331 & 0314413220 & $(1 ; 1,0,0,0)$ & \\
\hline 12 & 0011223344 & 0113243042 & 0410322143 & 0214402331 & 0312413420 & $(2 ; 1,1,0,0)$ & \\
\hline 13 & 0011223344 & 0113244032 & 0214302143 & 0312413420 & 0410422331 & $(4 ; 1,1,1,1)$ & perfect \\
\hline 14 & 0011223344 & 0110322443 & 0240231431 & 0320413412 & 0421421330 & $(2 ; 1,0,1,0)$ & \\
\hline
\end{tabular}

Table 2. Perfect decompositions of $K_{6,6}^{*}$.

\begin{tabular}{|c|c|c|c|c|c|c|c|}
\hline 1 & 001122334455 & 021021344553 & 011320354254 & 041225314350 & 031524324051 & 051423304152 & $(2 ; 1,0,1,0)$ \\
\hline 2 & 001122334455 & 021021344553 & 011324354250 & 031425324051 & 041520314352 & 051223304154 & $(4 ; 2,0,2,0)$ \\
\hline 3 & 001122334455 & 021021344553 & 014250351324 & 052043511432 & 031240542531 & 041541522330 & $(2 ; 1,0,1,0)$ \\
\hline 4 & 001122334455 & 021021354354 & 011520344253 & 041223314550 & 051324324051 & 031425304152 & $(24 ; 12,0,12,0)$ \\
\hline 5 & 001122334455 & 021021354354 & 031520344251 & 041325324150 & 051224314053 & 011423304552 & $(2 ; 1,0,1,0)$ \\
\hline 6 & 001122334455 & 021021354354 & 051420324153 & 011325344052 & 031524304251 & 041223314550 & $(12 ; 6,0,6,0)$ \\
\hline 7 & 001122334455 & 021021354354 & 052440325113 & 015315342042 & 041223314550 & 032514305241 & $(24 ; 6,6,6,6)$ \\
\hline 8 & 001122334455 & 021023344551 & 031425304152 & 041521324053 & 011324354250 & 051220314354 & $(12 ; 6,0,6,0)$ \\
\hline 9 & 001122334455 & 021023344551 & 041321354052 & 051224304153 & 031520314254 & 011425324350 & $(2 ; 1,0,1,0)$ \\
\hline 10 & 001122334455 & 021023344551 & 053041522413 & 013240532514 & 043521431250 & 035420311542 & $(2 ; 1,0,1,0)$ \\
\hline 11 & 001122334455 & 021023354154 & 052034425113 & 035045122431 & 041553213240 & 014314253052 & $(1 ; 1,0,0,0)$ \\
\hline 12 & 001122334455 & 021023354154 & 052440325113 & 035042251431 & 014315342052 & 041245532130 & $(2 ; 1,1,0,0)$ \\
\hline 13 & 001122334455 & 021024354153 & 051321304254 & 041523314052 & 031220344551 & 011425324350 & $(4 ; 2,0,2,0)$ \\
\hline 14 & 001122334455 & 021024354153 & 032132504514 & 052051133442 & 042315524031 & 014354122530 & $(2 ; 1,1,0,0)$ \\
\hline 15 & 001122334455 & 021024354351 & 042552411330 & 013253204514 & 053140541223 & 031521503442 & $(2 ; 1,0,1,0)$ \\
\hline 16 & 001122334455 & 021025314354 & 011552233440 & 032412503541 & 042051324513 & 052153143042 & $(1 ; 1,0,0,0)$ \\
\hline 17 & 001122334455 & 021320344551 & 033140251254 & 013542241053 & 052150431432 & 042352411530 & $(4 ; 2,0,2,0)$ \\
\hline 18 & 001122334455 & 021320344551 & 033142251054 & 013540241253 & 052350411432 & 042152431530 & $(4 ; 2,0,2,0)$ \\
\hline 19 & 001122334455 & 021320344551 & 033052254114 & 013523541240 & 051024314253 & 042150431532 & $(6 ; 3,0,3,0)$ \\
\hline 20 & 001122334455 & 021320344551 & 035425311240 & 014253351024 & 041541305223 & 052150431432 & $(2 ; 1,0,1,0)$ \\
\hline 21 & 001122334455 & 021320344551 & 035241351024 & 011254253043 & 052140531432 & 041542233150 & $(2 ; 1,0,1,0)$ \\
\hline 22 & 001122334455 & 021324354051 & 041520314253 & 031021344552 & 011425324350 & 051223304154 & $(36 ; 18,0,18,0)$ \\
\hline 23 & 001122334455 & 021324354051 & 041520314253 & 031425304152 & 051021324354 & 011223344550 & $(144 ; 36,36,36,36)$ \\
\hline 24 & 001122334455 & 021324354051 & 041520314253 & 031041542532 & 011445522330 & 051243502134 & $(36 ; 9,9,9,9)$ \\
\hline 25 & 001122334455 & 021324354051 & 052350311442 & 042145125330 & 013415522043 & 031025324154 & $(6 ; 3,0,3,0)$ \\
\hline 26 & 001122334455 & 021325304154 & 033442511520 & 014514235032 & 054310522431 & 041240213553 & $(2 ; 1,0,1,0)$ \\
\hline 27 & 001122334455 & 021325344150 & 041521304352 & 033551124024 & 054220531431 & 014510325423 & $(6 ; 3,0,3,0)$ \\
\hline 28 & 001122334455 & 021325344150 & 052052431431 & 011253354024 & 035421451032 & 041551304223 & $(2 ; 1,0,1,0)$ \\
\hline 29 & 001122334455 & 021420354153 & 011334422550 & 041531402352 & 031245243051 & 051043213254 & $(12 ; 6,0,6,0)$ \\
\hline 30 & 001122334455 & 021420354153 & 011350452432 & 053440231251 & 041552432130 & 033142251054 & $(12 ; 12,0,0,0)$ \\
\hline 31 & 001122334455 & 021420354153 & 011352452430 & 053140231254 & 041550432132 & 033442251051 & $(12 ; 6,0,6,0)$ \\
\hline 32 & 001122334455 & 021425314053 & 041523513042 & 031250243541 & 011334455220 & 051032432154 & $(4 ; 2,0,2,0)$ \\
\hline 33 & 001122334455 & 021524314053 & 041320354251 & 031025324154 & 051421304352 & 011223344550 & $(36 ; 18,0,18,0)$ \\
\hline 34 & 001122334455 & 021524314053 & 041320354251 & 031241502534 & 011445522330 & 051043542132 & $(36 ; 18,0,18,0)$ \\
\hline 35 & 001122334455 & 021024355143 & 041540532132 & 051254233041 & 011352452034 & 031442253150 & $(2 ; 1,0,1,0)$ \\
\hline 36 & 001122334455 & 021024355143 & 052053124134 & 035225144031 & 041321504532 & 011542542330 & $(2 ; 1,0,1,0)$ \\
\hline 37 & 001122334455 & 021024355143 & 013415235240 & 044513215032 & 031254253041 & 054220533114 & $(1 ; 1,0,0,0)$ \\
\hline 38 & 001122334455 & 021324355140 & 033150422514 & 013412452053 & 044115302352 & 054310325421 & $(4 ; 1,1,1,1)$ \\
\hline 39 & 001122334455 & 021423355140 & 052032411354 & 031531502442 & 043045125321 & 013410522543 & $(6 ; 3,0,3,0)$ \\
\hline 40 & 001122334455 & 021425305143 & 032032541541 & 043112452350 & 051334402152 & 013553104224 & $(12 ; 3,3,3,3)$ \\
\hline 41 & 001122334455 & 021425305143 & 044523311250 & 051354324021 & 031041355224 & 015320341542 & $(4 ; 1,1,1,1)$ \\
\hline 42 & 001122334455 & 021425315043 & 013445231052 & 054254133021 & 035140351224 & 043241155320 & $(2 ; 1,1,0,0)$ \\
\hline 43 & 001122334455 & 021024413553 & 042331154052 & 052043143251 & 014225135034 & 032154124530 & $(1 ; 1,0,0,0)$ \\
\hline 44 & 001122334455 & 021024453153 & 031520543241 & 051421304352 & 011325425034 & 041223355140 & $(2 ; 1,0,1,0)$ \\
\hline 45 & 001122334455 & 021324413550 & 031432452051 & 041540233152 & 051034214253 & 011225304354 & $(2 ; 1,0,1,0)$ \\
\hline 46 & 001122334455 & 021425403153 & 051243502134 & 044115233052 & 014532541320 & 031042243551 & $(2 ; 1,0,1,0)$ \\
\hline 47 & 001122334455 & 021425403153 & 051054324321 & 044113355220 & 013415235042 & 031251453024 & $(4 ; 1,1,1,1)$ \\
\hline 48 & 001122334455 & 021425403153 & 041352413520 & 053442231051 & 011550432432 & 033021451254 & $(6 ; 3,0,0,3)$ \\
\hline 49 & 001122334455 & 021425413053 & 043513204251 & 031245315024 & 011552233440 & 052143541032 & $(2 ; 1,0,1,0)$ \\
\hline 50 & 001122334455 & 021025415334 & 053142205413 & 044035231251 & 011552432430 & 035045142132 & $(1 ; 1,0,0,0)$ \\
\hline 51 & 001122334455 & 021325405431 & 014553341220 & 051421503243 & 031042513524 & 044115302352 & $(2 ; 1,0,1,0)$ \\
\hline 52 & 001122334455 & 021425405331 & 043512432150 & 031551304224 & 052034411352 & 014532541023 & $(2 ; 1,1,0,0)$ \\
\hline 53 & 001122334455 & 021425435130 & 014024351253 & 031521503442 & 041045522331 & 053241132054 & $(2 ; 1,0,1,0)$ \\
\hline 54 & 001122334455 & 021524415330 & 011435522043 & 032132451054 & 041350253142 & 054034231251 & $(2 ; 1,0,1,0)$ \\
\hline 55 & 001122334455 & 021421503543 & 041352402531 & 014510532432 & 052330411254 & 031551344220 & $(4 ; 2,0,2,0)$ \\
\hline 56 & 001122334455 & 021435215043 & 032542541031 & 014512205334 & 053024411352 & 042315513240 & $(12 ; 3,3,3,3)$ \\
\hline 57 & 001122334455 & 021051354324 & 031425304152 & 051354324021 & 014550341223 & 044253311520 & $(24 ; 12,0,0,12)$ \\
\hline 58 & 001122334455 & 021054354321 & 052440325113 & 014553341220 & 035241302514 & 042315315042 & $(24 ; 12,0,0,12)$ \\
\hline 59 & 001122334455 & 021351354024 & 014250341523 & 031425304152 & 044553311220 & 051054324321 & $(48 ; 12,12,12,12)$ \\
\hline
\end{tabular}

decompositions of $K_{6,6}$ are given in Table 3 with the notation $\left(M ; m_{1}, m_{2}\right)$ denoting that $|A u t(D)|=M$ and that there are $m_{1}$ mappings which preserve the bipartition and $m_{2}$ which reverse the bipartition.

3. Face 2-colourable triangulations of $\boldsymbol{K}_{\boldsymbol{n}, \boldsymbol{n}, \boldsymbol{n}}$. The principal method employed to tabulate face 2-colourable triangulations of $K_{n, n, n}$ was based on taking representatives 
Table 3. Hamiltonian decompositions of $K_{6,6}$.

$\begin{array}{lllll}1 & 001122334455 & 021324354051 & 041520314253 & (72 ; 36,36) \\ 2 & 001122334455 & 021425314053 & 043023154251 & (4 ; 4,0) \\ 3 & 001122334455 & 021524314053 & 041320354251 & (36 ; 36,0) \\ 4 & 001122334455 & 021425403153 & 043513205241 & (2 ; 1,1) \\ 5 & 001122334455 & 021425413053 & 043513204251 & (2 ; 2,0) \\ 6 & 001122334455 & 021423503541 & 043152402513 & (6 ; 6,0) \\ 7 & 001122334455 & 021351354024 & 014250341523 & (24 ; 12,12)\end{array}$

of the main classes of Latin squares of order $n$. For $4 \leq n \leq 7$ these are given in [3], and for $n=3$ there is just one main class. Having selected one such square, its triples are regarded as triangles with the common clockwise orientation (row, column, entry). In any biembedding containing this Latin square, the rotation about each point contains $n$ known ordered pairs; what remains unknown is the ordering of these pairs. By considering all possible orderings and rejecting those which give rise to pseudosurfaces, all biembeddings containing the given square may be determined. Working through the main classes of Latin squares of order $n$, each new biembedding was checked for isomorphism with those found previously. Finally, the results for $n \leq 6$ were reconciled with those of Section 2. For $n=7$ the large number of biembeddings to be checked required the use of an effective invariant in order to establish the isomorphism classes. The invariant used was as follows.

Consider a fixed embedding of $K_{n, n, n}$, and denote by $\rho_{u}$ the rotation around a vertex $u$. Since $\rho_{u}$ is a cyclic permutation, for each two neighbours $v$ and $w$ of $u$ there are $n_{1}$ and $n_{2}$ such that $w=\rho_{u}^{n_{1}}(v)$ and $w=\rho_{u}^{-n_{2}}(v)$ (where $1 \leq n_{1}, n_{2} \leq 2 n-1$ and $\left.n_{1}+n_{2}=2 n\right)$. Denote by $d(u ; v, w)$ the minimum of $n_{1}$ and $n_{2}$. Now if $d(u ; x, v)=1$ and $d(u ; v, y)=1, x \neq y$, then $d(v ; x, y)=2$. However if $d(u ; x, v)=3$ and $d(u ; v, y)=3$, $x \neq y$, then $d(v ; x, y)$ can be any even number from 2 to $n$. (The number $d(v ; x, y)$ is even because $x$ and $y$ belong to the same set of the tripartition.) For each vertex $v$, let $I_{v}$ be the sum of $2 n$ numbers given by

$$
I_{v}=\sum_{u}(d(v ; x, y): \text { where } d(u ; x, v)=d(u ; v, y)=3 \text { and } x \neq y),
$$

where the sum extends over all vertices $u$ of $K_{n, n, n}$ for which $u v$ is an edge. Then for $n=7,\left\{I_{v}: v \in V\left(K_{n, n, n}\right)\right\}$ is a satisfactory set of invariants.

For $n \leq 6$ we specify a representative biembedding from each isomorphism class by means of a vector $\left(i, j, p_{1}, p_{2}, p_{3}\right)$ where $i, j$ give the main class numbers of the two squares as in [3], and $p_{1}, p_{2}, p_{3}$ specify permutations applied respectively to the rows, columns and entries of the second square. From these the biembedding may be constructed as explained in the case $n=3$ below. We use $I$ to denote the identity permutation. In no case do we need to permute rows, columns and entries with each other. We also give information about the automorphism group of each biembedding $E$ with a second vector $\left(M ; m_{1}, m_{2}, m_{3}, m_{4}\right)$ denoting that $|A u t(E)|=M$ and that there are $m_{1}$ mappings which preserve orientation and colour classes, $m_{2}$ mappings which preserve orientation and reverse the colour classes, $m_{3}$ mappings which reverse orientation and preserve the colour classes, and $m_{4}$ mappings which reverse orientation and reverse the colour classes.

In the case $n=7$ the number of biembeddings is so large that it is only feasible to summarize the results in the form of tables showing which pairs of squares biembed and how many nonisomorphic biembeddings there are for a given pair. We also give a little more information about this case in Section 4. 
$\boldsymbol{n}=3$. There is just one biembedding given by $(1,1, I, I, 201),(108 ; 27$, $27,27,27)$. This biembedding is regular. To obtain the biembedding, take main class \#1 Latin square representative as

$$
\begin{array}{lll|}
\hline 0 & 1 & 2 \\
1 & 2 & 0 \\
2 & 0 & 1
\end{array} .
$$

This forms the first square. To form the second, apply the permutation $\left(\begin{array}{lll}0 & 1 & 2 \\ 2 & 0 & 1\end{array}\right)$ to the entries to get

$$
\begin{array}{lll|}
2 & 0 & 1 \\
0 & 1 & 2 \\
1 & 2 & 0
\end{array} .
$$

The rows and columns of each square are indexed by 0,1 and 2 . Then take the nine points of $K_{3,3,3}$ to be $0_{r}, 1_{r}, 2_{r}, 0_{c}, 1_{c}, 2_{c}, 0_{e}, 1_{e}, 2_{e}$. Black triangles with clockwise orientation $(r, c, e)$, are read from the first square so that, for example, the $(0,2)$ entry 2 gives the triangle $\left(0_{r}, 2_{c}, 2_{e}\right)$. White triangles with clockwise orientation $(r, e, c)$ are read from the second. The resulting rotation scheme is

$$
\begin{array}{lllllll}
0_{r}: & 0_{c} & 0_{e} & 1_{c} & 1_{e} & 2_{c} & 2_{e} \\
1_{r}: & 0_{c} & 1_{e} & 1_{c} & 2_{e} & 2_{c} & 0_{e} \\
2_{r}: & 0_{c} & 2_{e} & 1_{c} & 0_{e} & 2_{c} & 1_{e} \\
0_{c}: & 0_{e} & 0_{r} & 2_{e} & 2_{r} & 1_{e} & 1_{r} \\
1_{c}: & 0_{e} & 2_{r} & 2_{e} & 1_{r} & 1_{e} & 0_{r} \\
2_{c}: & 0_{e} & 1_{r} & 2_{e} & 0_{r} & 1_{e} & 2_{r} \\
0_{e}: & 0_{r} & 0_{c} & 1_{r} & 2_{c} & 2_{r} & 1_{c} \\
1_{e}: & 0_{r} & 1_{c} & 1_{r} & 0_{c} & 2_{r} & 2_{c} \\
2_{e}: & 0_{r} & 2_{c} & 1_{r} & 1_{c} & 2_{r} & 0_{c}
\end{array}
$$

This biembedding has its (full) automorphism group of order 108 with 27 automorphisms in each of the four classes described above. In this particular example, the embedding could equally well be specified by the vector $(1,1,120, I, I)$ since the second square may also be obtained from the first by applying the permutation $\left(\begin{array}{lll}0 & 1 & 2 \\ 1 & 2 & 0\end{array}\right)$ to the rows.

$\boldsymbol{n}=4$. There are two main classes of Latin squares but only one biembedding given by $(2,2, I, 3201, I),(192 ; 48,48,48,48)$. This biembedding is regular.

$\boldsymbol{n}=\mathbf{5}$. There are two main classes of Latin squares and three biembeddings.

1. $(1,1, I, 40123, I),(300 ; 75,75,75,75)$, regular,

2. $(1,1, I, 14023, I),(20 ; 5,5,5,5)$,

3. $(1,1,10234,30412,10234),(12 ; 3,3,3,3)$.

The second main class does not feature in any biembedding. 
Table 4. Biembeddings for $n=6$.

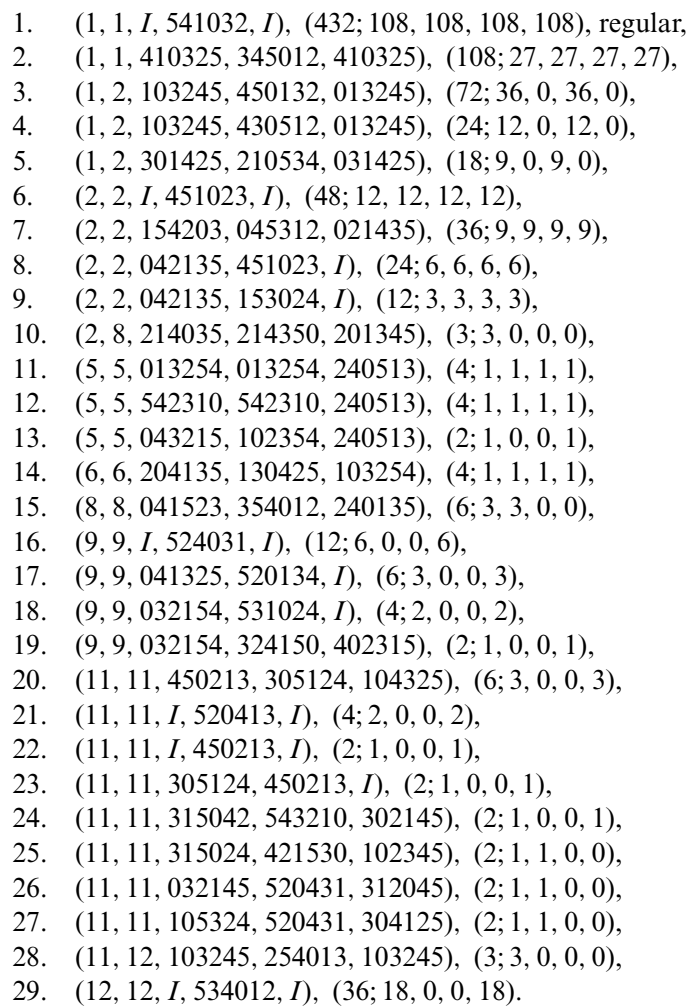

$\boldsymbol{n}=\mathbf{6}$. There are 12 main classes of Latin squares and 29 biembeddings. These are given in Table 4. Main classes 3, 4, 7 and 10 do not feature in any biembedding.

$\boldsymbol{n}=7$. There are 147 main classes of Latin squares and 23664 biembeddings. These are summarized in the 16 sub-tables of Table 5. The Latin squares partition into groups such that biembeddings only exist inside these groups. Each sub-table specifies the main class numbers of the squares along the top and left borders, and the entries in the body of each sub-table give the numbers of nonisomorphic embeddings. The number at the top left specifies the number of main classes included in that sub-table. It is interesting to note that inside each sub-table the number of zero entries (indicated by -) is small. Of the 23664 biembeddings, 4761 are biembeddings of a Latin square with itself. Although all 147 squares appear in Table 5, several squares do not biembed with themselves. Precisely one of the biembeddings is regular (see Section 4.2 for details).

\section{Remarks on the computational results.}

4.1. Reconciling the results of sections 2 and 3 . For each face 2-colourable triangulation of $K_{n, n, n}$ with $3 \leq n \leq 6$, three perfect decompositions of $K_{n, n}^{*}$ may 
be obtained as described in Proposition 2. For $n=3$, the three decompositions obtained from the unique biembedding given in section 3 are isomorphic with the unique perfect decomposition of $K_{n, n}^{*}$ given in section 2. The same is true for $n=4$. The decompositions of $K_{5,5}^{*}$ obtained by deleting respectively $R=\left\{0_{r}, 1_{r}, 2_{r}, 3_{r}, 4_{r}\right\}$, $C=\left\{0_{c}, 1_{c}, 2_{c}, 3_{c}, 4_{c}\right\}, E=\left\{0_{e}, 1_{e}, 2_{e}, 3_{e}, 4_{e}\right\}$ from each embedding of $K_{5,5,5}$ are shown in Table 6 with the embeddings numbered as in section 3, and the decompositions as in section 2 (Table 1). Note that each of the perfect decompositions of $K_{5,5}^{*}$ appears in precisely one row, as predicted by Proposition 2.

Table 5. Summary of $K_{7,7,7}$ results.

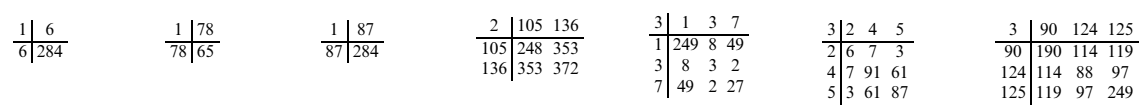
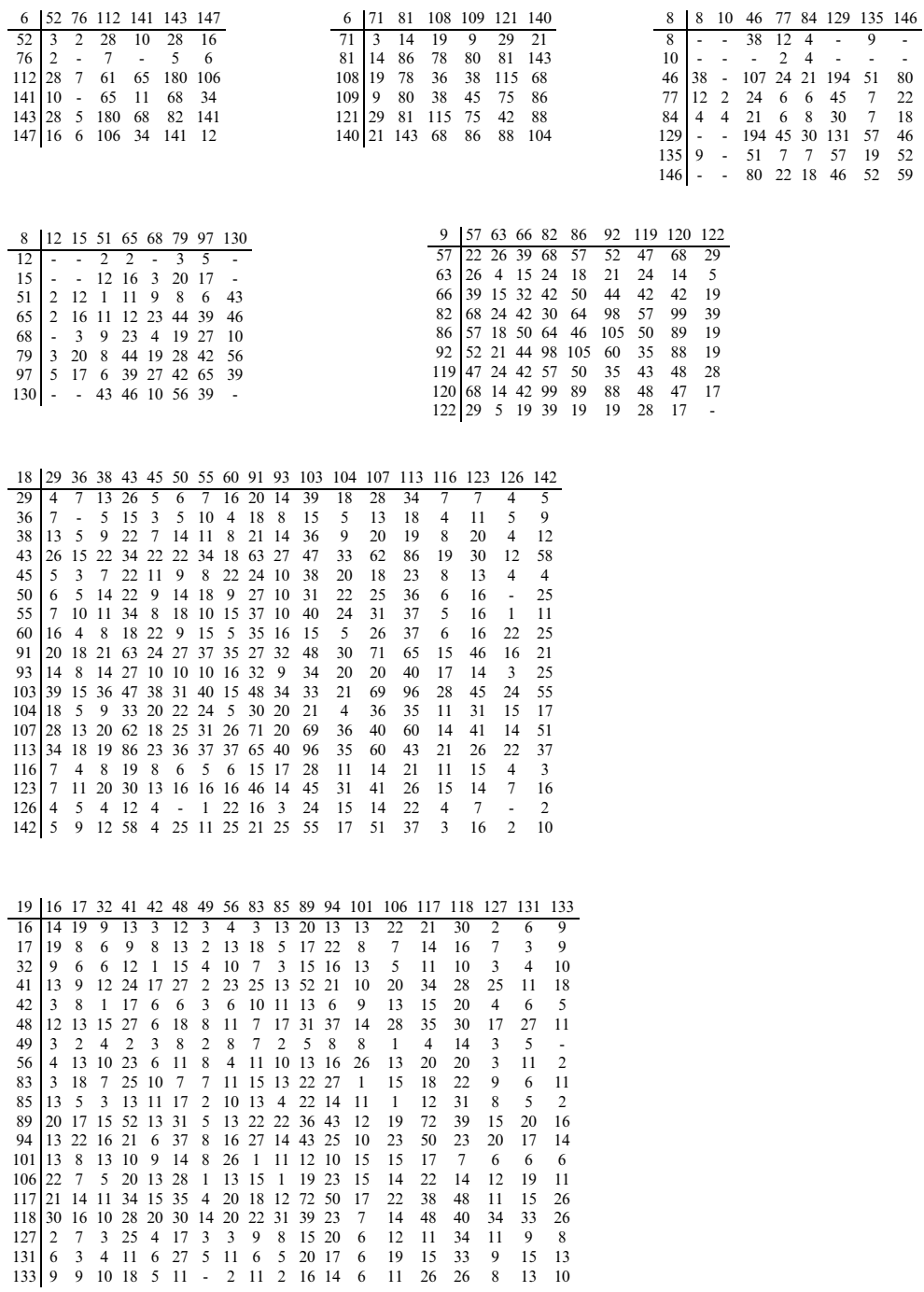
Table 5. Continued

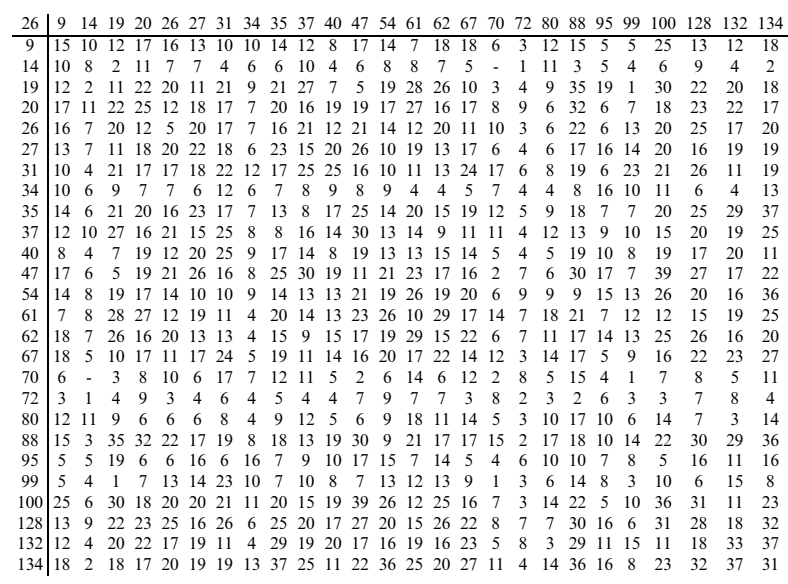

\begin{tabular}{|c|c|c|c|c|c|c|c|c|c|c|c|c|c|c|c|c|c|c|c|c|c|c|c|c|c|c|c|c|c|c|c|c|c|}
\hline 33 & 11 & & 18 & & & & & & & 30 & & & & & 50 & & 64 & & & & & & & & & & & & & & & & \\
\hline 11 & - & - & - & 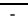 & - & - & 1 & - & 1 & - & - & - & - & - & - & 1 & - & & 7 & & 5 & 1 & 2 & 1 & 1 & 2 & 1 & 2 & - & - & & 2 & \\
\hline 13 & - & - & 3 & 3 & - & - & 1 & 1 & 5 & 1 & 4 & 2 & 3 & 1 & 1 & 2 & 4 & 4 & 3 & 1 & 1 & 8 & 6 & 1 & 5 & 1 & 5 & - & . & 3 & 1 & & 2 \\
\hline 18 & - & 3 & 4 & 6 & - & 3 & 9 & 1 & 3 & 4 & 18 & 8 & 22 & 3 & 6 & 8 & 6 & 4 & 4 & 2 & 4 & 21 & 16 & 10 & 8 & 1 & 18 & - & & 4 & & 15 & \\
\hline 21 & - & 3 & 6 & 12 & 3 & 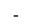 & 7 & - & 2 & 19 & 14 & 12 & 22 & 4 & 9 & 9 & 7 & 13 & 3 & & 2 & 26 & 14 & 9 & 3 & 4 & 23 & 2 & 1 & 10 & 6 & 8 & \\
\hline 22 & - & - & - & 3 & 6 & 2 & 2 & - & - & 6 & 11 & 2 & 7 & 2 & 3 & 2 & 1 & - & 1 & 4 & 1 & 15 & 9 & 4 & 5 & 7 & 8 & 1 & & 5 & 3 & 1 & 2 \\
\hline 23 & - & - & 3 & - & 2 & 4 & 4 & 4 & 2 & - & 1 & 4 & 2 & - & 2 & 3 & - & 3 & 7 & & 7 & 5 & 5 & 3 & 7 & 1 & 12 & 3 & & & & & \\
\hline 24 & 1 & 1 & 9 & 7 & 2 & 4 & 2 & - & - & 11 & 27 & 5 & 19 & 1 & 5 & 5 & 11 & 6 & 1 & 1 & - & 31 & 17 & 5 & 1 & 2 & 15 & 2 & & 4 & 1 & 5 & \\
\hline 25 & - & 1 & 1 & - & - & 4 & - & - & - & 1 & 2 & - & 1 & - & 4 & 1 & 2 & - & - & 1 & - & - & & - & . & 1 & 1 & - & & 1 & 5 & 1 & - \\
\hline 28 & 1 & 5 & 3 & 2 & - & 2 & - & - & 2 & 4 & 3 & 1 & 7 & 2 & 6 & 7 & 2 & 2 & 9 & 2 & 6 & 28 & 13 & 4 & 2 & 3 & 11 & 8 & & 4 & 2 & & \\
\hline 30 & - & 1 & 4 & 19 & 6 & - & 11 & 1 & 4 & 8 & 16 & 6 & 5 & - & 1 & 7 & 8 & 15 & 5 & & 3 & 17 & 19 & 8 & 10 & 1 & 24 & 1 & & 7 & 3 & & 4 \\
\hline 33 & - & 4 & 18 & 14 & 11 & 1 & 27 & 2 & 3 & 16 & 30 & 18 & 17 & 1 & 11 & 22 & 19 & 15 & 9 & 5 & 4 & 30 & 37 & 17 & 24 & 4 & 42 & 3 & 3 & 15 & 10 & 18 & 4 \\
\hline 39 & - & 2 & 8 & 12 & 2 & 4 & 5 & - & 1 & 6 & 18 & 6 & 20 & 1 & 13 & 3 & 9 & 3 & 2 & & 1 & 18 & 24 & 4 & 4 & 2 & 24 & 4 & & . & 3 & 12 & \\
\hline 44 & - & 3 & 22 & 22 & 7 & 2 & 19 & 1 & 7 & 5 & 17 & 20 & 21 & 4 & 22 & 19 & 16 & 20 & 7 & 2 & 1 & 23 & 35 & 8 & 11 & 5 & 36 & 9 & & 12 & 2 & 11 & 5 \\
\hline 53 & - & 1 & 3 & 4 & 2 & - & 1 & - & 2 & - & 1 & 1 & 4 & 2 & 2 & 1 & - & 1 & - & 2 & - & 6 & 2 & 1 & & 1 & & - & & - & 5 & & - \\
\hline 58 & - & 1 & 6 & 9 & 3 & 2 & 5 & 4 & 6 & 1 & 11 & 13 & 22 & 2 & 11 & 5 & 8 & 2 & 4 & & 3 & 24 & 18 & 7 & 12 & - & 16 & 5 & & 8 & 5 & 12 & 2 \\
\hline 59 & 1 & 2 & 8 & 9 & 2 & 3 & 5 & 1 & 7 & 7 & 22 & 3 & 19 & 1 & 5 & 8 & 10 & 7 & 7 & 4 & 1 & 20 & 24 & 10 & 14 & 2 & 14 & 2 & & 12 & 2 & 13 & 3 \\
\hline 64 & . & 4 & 6 & 7 & 1 & - & 11 & 2 & 2 & 8 & 19 & 9 & 16 & - & 8 & 10 & 12 & 13 & - & & 3 & 16 & 20 & 13 & 11 & 2 & 19 & 4 & & & 4 & 12 & \\
\hline 69 & - & 4 & 4 & 13 & - & 3 & 6 & - & 2 & 15 & 15 & 3 & 20 & 1 & 2 & 7 & 13 & 3 & 3 & 4 & 2 & 20 & 18 & 11 & 3 & 5 & 11 & 2 & & 7 & 5 & & 2 \\
\hline 73 & 7 & 3 & 4 & 3 & 1 & 7 & 1 & - & 9 & 5 & 9 & 2 & 7 & - & 4 & 7 & - & 3 & 5 & 3 & 3 & 14 & 7 & 5 & & 1 & & 1 & & & 3 & & 3 \\
\hline 74 & - & 1 & 2 & 3 & 4 & 1 & 1 & 1 & 2 & - & 5 & 1 & 2 & 2 & 2 & 4 & 3 & 4 & 3 & 2 & - & 10 & 1 & 3 & 3 & 1 & & & & 5 & 2 & & 1 \\
\hline 75 & 5 & 1 & 4 & 2 & 1 & 7 & - & - & 6 & 3 & 4 & 1 & 1 & - & 3 & 1 & 3 & 2 & 3 & - & 4 & 6 & 7 & 3 & 1 & 1 & 1 & 1 & & 4 & 4 & 2 & - \\
\hline 96 & 1 & 8 & 21 & 26 & 15 & 5 & 31 & - & 28 & 17 & 30 & 18 & 23 & 6 & 24 & 20 & 16 & 20 & 14 & 10 & 6 & 63 & 49 & 34 & 11 & 25 & 45 & 6 & & 17 & & & 7 \\
\hline 98 & 2 & 6 & 16 & 14 & 9 & 5 & 17 & - & 13 & 19 & 37 & 24 & 35 & 2 & 18 & 24 & 20 & 18 & 7 & 1 & 7 & 49 & 24 & 21 & 21 & 9 & 50 & 7 & & 12 & 1 & 28 & 13 \\
\hline 102 & 1 & 1 & 10 & 9 & 4 & 3 & 5 & - & 4 & 8 & 17 & 4 & 8 & 1 & 7 & 10 & 13 & 11 & 5 & 3 & 3 & 34 & 21 & 18 & 11 & 2 & 27 & 4 & & 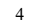 & 4 & 13 & 8 \\
\hline 11 & 1 & 5 & 8 & 3 & 5 & 7 & 1 & _ & 2 & 10 & 24 & 4 & 11 & 2 & 12 & 14 & 11 & 3 & 2 & 3 & 1 & 11 & 21 & 11 & 10 & & 12 & 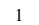 & & & & 15 & \\
\hline 111 & 2 & 1 & 1 & 4 & 7 & 1 & 2 & 1 & 3 & 1 & 4 & 2 & 5 & 1 & - & 2 & 2 & 5 & 1 & 1 & 1 & 25 & 9 & 2 & 3 & 1 & 10 & 2 & & 6 & 2 & 4 & 1 \\
\hline 114 & 1 & 5 & 18 & 23 & 8 & 12 & 15 & 1 & 11 & 24 & 42 & 24 & 36 & 4 & 16 & 14 & 19 & 11 & 2 & 6 & 1 & 45 & 50 & 27 & 12 & 10 & 43 & 3 & & 13 & 7 & 28 & 2 \\
\hline 11 & 2 & . & - & 2 & 1 & 3 & 2 & . & 8 & 1 & 3 & 4 & 9 & & 5 & 2 & 4 & 2 & 1 & 2 & 1 & 6 & 7 & 4 & 1 & 2 & 3 & 3 & & & & & \\
\hline 137 & - & - & - & 1 & - & - & 2 & - & - & - & 3 & 2 & 4 & 4 & - & 1 & 3 & 1 & 4 & 1 & 3 & 6 & 7 & 1 & 3 & - & 4 & - & - & 2 & 1 & 6 & 4 \\
\hline 13 & & 3 & 4 & 10 & 5 & 2 & 4 & 1 & 4 & 7 & 15 & 1 & 12 & & 8 & 12 & 6 & & 5 & & & 17 & 12 & 4 & & & 13 & & & & & & \\
\hline 138 & - & 1 & - & 6 & 3 & - & 1 & 5 & 2 & 3 & 10 & 3 & 2 & 5 & 5 & 2 & 4 & 5 & 3 & 2 & 4 & 6 & 1 & 4 & 1 & 2 & 7 & 4 & & 3 & & 1 & 2 \\
\hline 144 & 2 & - & 15 & 8 & 1 & 4 & 5 & 1 & 6 & 7 & 18 & 12 & 11 & 4 & 12 & 13 & 12 & 8 & 2 & 3 & 2 & 30 & 28 & 13 & 15 & 4 & 28 & 3 & & 8 & 1 & 18 & 5 \\
\hline 145 & & 2 & - & 8 & 2 & 2 & 2 & & 1 & 4 & 4 & 3 & 5 & & 2 & 3 & 4 & 2 & 3 & 1 & & 7 & & 8 & 1 & 1 & 2 & 2 & & - & & 5 & \\
\hline
\end{tabular}

Table 7 gives the corresponding results for $n=6$ with the embeddings numbered as in section 3 (Table 4), and the decompositions as in section 2 (Table 2). Again note that each of the perfect decompositions of $K_{6,6}^{*}$ appears in precisely one row, as predicted by Proposition 2.

4.2. Regular biembeddings. Our computational results show that for each $n \in$ $\{3,4,5,6,7\}$ there exists precisely one regular face 2-colourable triangular embedding of $K_{n, n, n}$. It will be shown in [4] that this uniqueness result in fact holds for every $n$. In each case this embedding consists of a biembedding of two cyclic Latin squares of order $n$. Such biembeddings are not new. They may be constructed for each $n \geq 3$ directly from cyclic Latin squares or from voltage graphs. The former approach has the advantage of easily establishing the regularity of these embeddings. Take two 
BIEMBEDDINGS OF LATIN SQUARES

Table 6. $K_{5,5,5}$ and $K_{5,5}^{*}$ correspondence.

\begin{tabular}{cccc}
\hline & \multicolumn{3}{c}{ Deleted set and $K_{5,5}^{*}$ decomposition } \\
\cline { 2 - 4 }$K_{5,5,5}$ embedding & $R$ & $C$ & $E$ \\
\hline 1 & 4 & 4 & 4 \\
2 & 7 & 6 & 7 \\
3 & 13 & 13 & 13 \\
\hline
\end{tabular}

Table 7. $K_{6,6,6}$ and $K_{6,6}^{*}$ correspondence.

\begin{tabular}{cccc}
\hline & \multicolumn{3}{c}{ Deleted set and $K_{6,6}^{*}$ decomposition } \\
\cline { 2 - 4 }$K_{6,6,6}$ embedding & $R$ & $C$ & $E$ \\
\hline 1 & 23 & 23 & 23 \\
2 & 24 & 24 & 24 \\
3 & 57 & 57 & 57 \\
4 & 30 & 58 & 30 \\
5 & 48 & 48 & 48 \\
6 & 4 & 59 & 4 \\
7 & 56 & 56 & 56 \\
8 & 7 & 31 & 31 \\
9 & 27 & 27 & 40 \\
10 & 43 & 43 & 43 \\
11 & 35 & 35 & 38 \\
12 & 1 & 1 & 47 \\
13 & 21 & 53 & 26 \\
14 & 28 & 28 & 41 \\
15 & 52 & 52 & 52 \\
16 & 6 & 29 & 8 \\
17 & 25 & 19 & 39 \\
18 & 18 & 55 & 17 \\
19 & 51 & 46 & 20 \\
20 & 15 & 15 & 15 \\
21 & 13 & 32 & 2 \\
22 & 5 & 49 & 9 \\
23 & 36 & 54 & 10 \\
24 & 44 & 45 & 3 \\
25 & 14 & 37 & 37 \\
26 & 50 & 12 & 50 \\
27 & 11 & 11 & 42 \\
28 & 16 & 16 & 16 \\
29 & 22 & 34 & 33 \\
\hline
\end{tabular}

isomorphic squares $L_{1}$ and $L_{2}$ whose rows, columns and entries are indexed by the group $\mathbb{Z}_{n}$ and whose entries in row $i$, column $j$ are given respectively by $L_{1}(i, j) \equiv$ $i+j(\bmod n)$ and $L_{2}(i, j) \equiv i+j-1(\bmod n)$. Proceeding as in the $n=3$ case of Section 3 , it is easy to see that we may obtain a rotation scheme for a biembedding of $L_{1}$ and $L_{2}$. Furthermore, this biembedding has $n^{2}$ automorphisms of the form $\phi_{\alpha, \beta}:\left(i_{r}, j_{c}, k_{e}\right) \rightarrow\left((i+\alpha)_{r},(j+\beta)_{c},(k+\alpha+\beta)_{e}\right)$, and these all preserve the colour classes, the orientation, and the rows, columns and entries. In addition, the mapping $\chi:\left(i_{r}, j_{c}, k_{e}\right) \rightarrow\left(i_{c},-j_{e},-k_{r}\right)$ gives an automorphism of order 3 which permutes rows, columns and entries, but preserves the colour classes and the orientation. The mapping $\mu:\left(i_{r}, j_{c}, k_{e}\right) \rightarrow\left(i_{c}, j_{r}, k_{e}\right)$ gives an automorphism of order 2 which preserves the colour classes but reverses orientation, and the mapping $v:\left(i_{r}, j_{c}, k_{e}\right) \rightarrow\left(-i_{c},-j_{r},(-k-1)_{e}\right)$ 


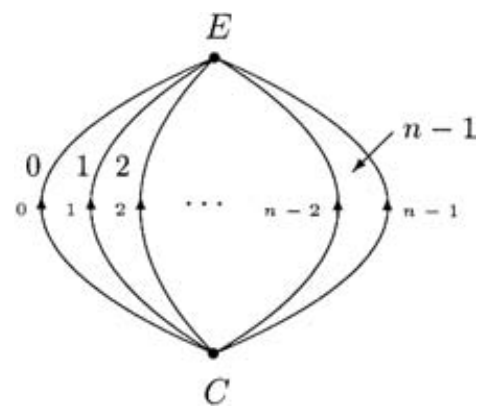

Figure 1 Voltage graph for regular biembedding.

gives an automorphism of order 2 which reverses the colour classes but preserves the orientation. It follows that the group of automorphisms generated by these mappings has order at least $12 n^{2}$. Since this is the maximum possible order, we deduce that this group is the full automorphism group of the biembedding and that the biembedding is regular.

An alternative description given in [11] uses the voltage graph shown in Figure 1. The graph has two vertices labelled $C$ and $E$ connected by $n$ arcs directed from $C$ to $E$ and labelled with elements of the group $\mathbb{Z}_{n}$ in order, as shown. The resulting regions are labelled 0 to $n-1$, with region $i$ bordered by the arcs labelled $i-1$ and $i$, as shown. Clockwise rotations are imposed at $C$ and $E$. The lifted graph has $2 n$ vertices which may be taken as $C_{0}, C_{1}, \ldots, C_{n-1}, E_{0}, E_{1}, \ldots, E_{n-1}$, and $n$ regions $r_{0}, r_{1}, \ldots, r_{n-1}$ whose clockwise boundaries are given by

$$
\begin{array}{rccccccc}
r_{0}: & C_{0} & E_{0} & C_{1} & E_{1} & \ldots & C_{n-1} & E_{n-1} \\
r_{1}: & C_{0} & E_{1} & C_{1} & E_{2} & \ldots & C_{n-1} & E_{0} \\
\vdots & & & & & \vdots & & \\
r_{n-1}: & C_{0} & E_{n-1} & C_{1} & E_{0} & \ldots & C_{n-1} & E_{n-2}
\end{array}
$$

Inserting a vertex $R_{i}$ into the interior of each region $r_{i}$ and joining it with new nonintersecting edges to all the vertices on the boundary of $r_{i}$ gives the partial rotation scheme (rotations about $R_{i}$ )

$$
\begin{array}{rccccccc}
R_{0}: & C_{0} & E_{0} & C_{1} & E_{1} & \ldots & C_{n-1} & E_{n-1} \\
R_{1}: & C_{0} & E_{1} & C_{1} & E_{2} & \ldots & C_{n-1} & E_{0} \\
\vdots & & & & & \vdots & & \\
R_{n-1}: & C_{0} & E_{n-1} & C_{1} & E_{0} & \ldots & C_{n-1} & E_{n-2}
\end{array}
$$

It is then a routine matter to complete this scheme (rotations about $C_{i}$ and $E_{i}$ ) and to verify that it gives the same biembedding as the previous method (by mapping $R_{i}$ to $i_{r}$, etc.).

The paper [4] gives a systematic investigation of voltage graphs similar to that shown in Figure 1, but with varying distributions of the voltages. An alternative voltage graph construction for the dual of the regular biembeddings is given in [7]. 
4.3. Recursive constructions. In [7] a variety of recursive constructions for embeddings are presented. These require, as a basic ingredient, face 2-colourable triangulations of $K_{n, n, n}$ in which there are parallel classes of triangular faces in one, or preferably both, of the two colour classes. By a parallel class we mean a set of vertexdisjoint triangular faces whose vertices collectively cover the complete vertex set of the graph $K_{n, n, n}$. Such a set of triangles forms a 2-factor of the graph and is referred to as a patchwork by Gross and Tucker [9, p. 155]. Since the regular embeddings described above and used in [7] involve cyclic Latin squares, these embeddings do indeed have parallel classes, in both colour classes, whenever $n$ is odd because the Latin squares have transversals. But cyclic Latin squares of even order do not possess transversals and so the resulting regular embeddings do not have parallel classes when $n$ is even. However, by examining the biembeddings listed in Section 3 for $n=6$ we can identify suitable triangulations of $K_{6,6,6}$, such as \#6 which biembeds two representatives of main class \#2. This Latin square has 32 transversals and so triangulation \#6 has 32 parallel classes of triangular faces in each of the two colour classes. By using this triangulation, we may extend the results of [7] significantly. For example, we may give a non-orientable version of Constructions 4 and 5 (alluded to in the Concluding Remarks) of that paper but now with $m=6$ or, by making use of a slight generalization of Construction 2, with $m=6^{r} s$ where $r>0$ and $s$ is odd.

Construction. Suppose that $n \equiv 1$ or $3(\bmod 6)$ with $n \geq 7$. Suppose also that we have $k$ differently labelled face 2-colourable triangulations of $K_{m, m, m}$, where $m=6^{r} s$, $r>0$ and $s$ is odd, all of which have a common parallel class of black triangular faces. Then we may construct $k^{(n-1)(n-3) / 6}$ differently labelled face 2-colourable non-orientable triangulations of $K_{m(n-1)+1}$.

By saying that two embeddings are "differently labelled" we mean that there is a face boundary in one embedding that is not a face boundary in the other even though the underlying graphs are identical. The proof follows the discussion in [7] but needs as ingredients a face 2-colourable triangulation of $K_{n}$ (either orientable or nonorientable) and a face 2-colourable triangulation of $K_{2 m+1}$ (necessarily nonorientable). These ingredients may be found in $[8,10,12]$.

4.4. Automorphisms of $K_{7,7,7}$ embeddings. Observe that all the face 2-colourable triangulations of $K_{n, n, n}$, where $3 \leq n \leq 5$, have automorphism group of order at least $n$. As regards the 29 face 2-colourable triangulations of $K_{6,6,6}$, exactly 14 of them have $|A u t(E)| \geq 6$. However, automorphism groups of order at least 7 are rare among the face 2-colourable triangulations of $K_{7,7,7}$. Whenever $|A u t(E)| \geq 7$, then the Latin squares involved in the biembedding are either \#3 (the square generated by the STS(7)) or \#7 (the cyclic square) of [3]. As there are only 32 biembeddings in which only \#3 and \#7 appear (see Table 5), we list all these embeddings in Table 8, in the same format as those of $K_{6,6,6}$ in Table 4.

Among these biembeddings, only \#4 and \#6 are vertex-transitive. The 12 embeddings \#7-\#18 are not transitive on vertices, but they can be obtained by the voltage graph construction, assigning voltages to a dipole. However, the voltages assigned are different from those depicted on Figure 1. We discuss these embeddings in [4]. 
Table 8. Biembeddings of \#3 and \#7.

1. $(3,3,1603452,5416023,2031456),(28 ; 7,7,7,7)$,

2. $(3,3,1560234,1560234,3012456),(4 ; 1,1,1,1)$,

3. $(3,3,2651304,6013452,3120456),(1 ; 1,0,0,0)$,

4. $(3,7,4253610,0231456,0421356),(21 ; 21,0,0,0)$,

5. $(3,7,5142360,0351426,2403156),(3 ; 3,0,0,0)$,

6. $(7,7,1234560, I, I),(588 ; 147,147,147,147)$, regular

7. $(7,7,1256340, I, I),(28 ; 7,7,7,7)$,

8. $(7,7,1534620, I, I),(28 ; 7,7,7,7)$,

9. $(7,7,1436520, I, I),(28 ; 7,7,7,7)$,

10. $(7,7,1564230, I, I),(28 ; 7,7,7,7)$,

11. $(7,7,1352640, I, I),(28 ; 7,7,7,7)$,

12. $(7,7,1235640, I, I),(28 ; 7,7,7,7)$,

13. $(7,7,1456230, I, I),(28 ; 7,7,7,7)$,

14. $(7,7,5246310, I, I),(14 ; 7,0,7,0)$,

15. $(7,7,4265310, I, I),(14 ; 7,0,7,0)$,

16. $(7,7,1536240, I, I),(14 ; 7,0,7,0)$,

17. $(7,7,1254630, I, I),(14 ; 7,0,7,0)$,

18. $(7,7,1546320, I, I),(14 ; 7,0,7,0)$,

19. $(7,7,5341260,0534126,4230156),(12 ; 3,3,3,3)$,

20. $(7,7,1253460,0234516,0134256),(12 ; 3,3,3,3)$,

21. $(7,7,4512360,0451236,3401256),(12 ; 3,3,3,3)$,

22. $(7,7,5612340,0312456,1203456),(6 ; 3,0,3,0)$,

23. $(7,7,1425360,0142536,0314256),(6 ; 3,0,3,0)$,

24. $(7,7,5234610,0135426,3042156),(4 ; 1,1,1,1)$,

25. $(7,7,4532610,0145236,0341256),(4 ; 1,1,1,1)$,

26. $(7,7,5234610,0145236,0341256),(4 ; 1,1,1,1)$,

27. $(7,7,5613420,0251346,1402356),(2 ; 1,0,1,0)$,

28. $(7,7,5461230,0421356,2130456),(2 ; 1,0,1,0)$,

29. $(7,7,3456120,0234516,1234056),(2 ; 1,0,1,0)$,

30. (7, 7, 2361540, 0534126, 4230156), (2; 1, 0, 1, 0),

31. $(7,7,2456130,0235416,1243056),(2 ; 1,0,0,1)$,

32. $(7,7,4623510,0235146,1240356),(2 ; 1,0,1,0)$.

Table 9. Embeddings by order of automorphism group.

\begin{tabular}{cc}
\hline $\mid$ Aut $(E) \mid$ & number of embeddings \\
\hline 1 & 22114 \\
2 & 1270 \\
3 & 166 \\
4 & 59 \\
6 & 37 \\
$\geq 7$ & 18 \\
\hline
\end{tabular}

To complete the information, in Table 9 we present the numbers of biembeddings according to the order of $\operatorname{Aut}(E)$.

4.5. Biembeddings of Latin squares. Our computational results show that not every main class of Latin square features in a biembedding. For $n \leq 6$, those that do not are the Cayley table of the Klein group of order 4, the Latin square of order 5 which does not come from the cyclic group, and four of the 12 main classes of Latin square of order 6. In fact, the first of these exceptions can easily be established 
by hand calculation. However, for $n=7$ all the main classes of Latin square feature in biembeddings. It is a very interesting question whether the six small exceptions identified above are the only ones.

More startling are the partitioning results given in Table 5 for the case $n=7$, namely that the 147 main classes of Latin square partition into 16 subsets respectively containing 1, 1, 1, 2, 3, 3, 3, 6, 6, 8, 8, 9, 18, 19, 26 and 33 classes such that the biembeddings of Latin squares exist only when both squares belong to the same subset of the partition. Similar partitioning can be identified for $n=6$ and, trivially, for $n=$ 3,4 and 5. In our opinion this is the most interesting and perhaps unexpected property to emerge from our computations. It raises the problem of providing a mathematical explanation for these results. We hope to return to this in a future paper.

Acknowledgement. This work was done during visits by the first two authors to the Slovak University of Technology and by the third author to the Open University. We thank each university for its hospitality and the Leverhulme Trust for financial support under grant F/00269/E. We also thank the referee for helpful comments.

\section{REFERENCES}

1. D. Archdeacon, Topological graph theory - a survey, Congr. Numer. 115 (1996), 5-54.

2. C. P. Bonnington, M. J. Grannell, T. S. Griggs and J. Širáň, Exponential families of non-isomorphic triangulations of complete graphs, J. Combin. Theory B 78 (2000), 169-184.

3. C. J. Colbourn and J. H. Dinitz (eds.), CRC Handbook of Combinatorial Designs (CRC Press, Boca Raton, 1996).

4. M. J. Grannell, T. S. Griggs, M. Knor and J. Širáň, Triangulations of orientable surfaces by complete tripartite graphs, submitted.

5. M. J. Grannell, T. S. Griggs and J. Širáň, Face 2-colourable triangular embeddings of complete graphs, J. Combin. Theory B 74 (1998), 8-19.

6. M. J. Grannell, T. S. Griggs and J. Širáň, Surface embeddings of Steiner triple systems, J. Combin. Des. 6 (1998), 325-336.

7. M. J. Grannell, T. S. Griggs and J. Širán̆, Recursive constructions for triangulations, J. Graph Theory 39 (2002), 87-107.

8. M. J. Grannell and V. P. Korzhik, Nonorientable biembeddings of Steiner triple systems, Discrete Math., to appear.

9. J. L. Gross and T. W. Tucker, Topological graph theory (John Wiley, 1987).

10. G. Ringel, Map color theorem (Springer-Verlag, 1974).

11. S. Stahl and A. T. White, Genus embeddings for some complete tripartite graphs, Discrete Math. 14 (1976), 279-296.

12. J. W. T. Youngs, The mystery of the Heawood conjecture, in: Graph theory and its applications (B. Harris, ed.), (Academic Press, 1970), 17-50. 